\title{
Considering sensor characteristics during measurement- system design for structural system identification
}

\author{
James-A. Goulet ${ }^{1}$, Prakash Kripakaran ${ }^{1}$ and Ian F. C. Smith ${ }^{1}$ \\ ${ }^{1}$ IMAC, Applied Computing and Mechanics Laboratory \\ EPFL, École Polytechnique Fédérale de Lausanne \\ CH-1015, Lausanne, Switzerland, \\ Email: James.Goulet@epfl.ch
}

\begin{abstract}
This paper presents a method for measurement-system design through criteria related to model based structural identification. Using a multi-model approach and results from previous research carried out at EPFL, an improved algorithm is proposed. The algorithm accounts for various types of sensors having different accuracies and taking different kinds of measurements. The algorithm selects sensor types and locations that minimise the number of non-identified candidate models. The results show that the approach provides an alternative to selecting and placing sensors using engineering experience alone, and that a scientific approach based on sensor characteristics and modelling error is feasible. A single span composite bridge is used to illustrate the algorithm. It is shown that adding more than 9 sensors, from a possible set of 34, will not provide further useful information for structural identification.
\end{abstract}

\section{Introduction}

Decisions to initiate measurement studies and then subsequent choices related to the types of sensors, including their locations, are almost exclusively based on engineering judgement. Engineering judgement however may not adequately assess all important factors and as a result, measurement tasks may not provide useful information. More systematic approaches assist in the configuration of measurement systems that gather data to support decision-making for structural management. Performance metrics that allow engineers to compare different measurement systems are required. Since the cost of sensors is decreasing there is a trend towards placing large numbers of sensors to monitor structures. Although, the cost of sensors may now be low, the cost associated with making sense of the data is not. Making effective measurement choices from the beginning could enable engineers to focus on the identification task without being "drowned" in insignificant data. 
A systematic approach to interpretation of measurement data employs methodologies developed in the field of system identification (Ljung 1999). System identification can be defined as the task of determining the state of a system by comparing model predictions with measurements. Since system identification is an inverse engineering approach which is solved in an open world, many models may predict the same measured behaviour. Raphael and Smith (2003) stated that errors coming from model and measurements may compensate each other, leading to the identification of wrong candidate behaviour models. To overcome this difficulty, Raphael and Smith (1998) proposed a multi-model approach, which involved the generation of thousands of possible behaviour scenarios. From there, candidate models were selected by comparing models to measured data. The approach has been successfully applied to simulated and laboratory cases, using static measurements (Robert-Nicoud et al. 2005a; Robert-Nicoud et al. 2005c; Saitta et al. 2005; Saitta et al. 2006). Other work has also been done in the field of system identification using static measurements (Banan and Hjelmstad 1994a; Banan and Hjelmstad 1994b; Bell et al. 2007; Jang et al. 2002; Sanayei et al. 2006; Sanayei et al. 1997; Sanayei and Saletnik 1996; Yeo et al. 2000). Most other proposals for system identification are based on dynamic measurements and they involve refinement of a single model (Beck and Katafygiotis 1998; Bell et al. 2007; Catbas et al. 2008; Katafygiotis and Beck 1998; Morassi and Tonon 2008; Nowak and Cho 2007; Sanayei et al. 2006).

Although the challenge of placing sensors has already been studied by other researchers (Kang et al. 2008; Meo and Zumpano 2005), proposals are limited to the scope of updating models using dynamic measurements. Previous research at EPFL in measurement system design has lead to greedy and global optimization strategies to place sensors (Kripakaran et al. 2007a; Kripakaran et al. 2007b; Robert-Nicoud et al. 2005). A greedy strategy supports system identification using iterative placementmeasurement-interpretation cycles while the global optimization approach supports the configuration of initial measurement systems most effectively. All of these studies focused on identifying the best measurement locations for a single type of sensor. However, there are many types of sensors that are currently available to measure a range of response types. Sensors differ in their technology, accuracy and cost. Engineers need rational approaches that help determine optimal measurement locations and sensor types.

Building on previous research, this paper presents a rational and systematic method for measurement system design considering multiple sensor characteristics. Measurement system design focuses on reducing the number of candidate behaviour models using recent research into multiple-model structural identification and advanced computing methods. The following sections describe the sensor selection algorithm. Important aspects of the method are then illustrated using a case study. 


\section{Sensor Placement Algorithm}

Sensor-data driven decision support systems can be a basis for proactive management of structural facilities. Sensor information has the potential to improve the performance and reduce the life-cycle cost over a broad range of infrastructure systems (Domer et al. 2003; Garrett et al. 2006) For effective decision support, it is essential that sensor systems are configured to measure responses at locations where the measured data can be meaningfully interpreted. Robert-Nicoud et al. (2005) developed a sensor placement methodology that consists of two steps - (1) generation of candidate models and (2) sensor performance evaluation at each location. Since generating all possible models is combinatorial, a sample population of models is selected using assumptions made by the engineer.

To generate models that include damage scenarios, the Monte Carlo method is used via the Latin-Hypercube sampling algorithm. Each model is evaluated by finite element analysis. Its predictions $p_{i}$ corresponding to each sensor type are computed at all possible sensor locations and then stored in a set $M_{0}$. The number of sampled models $N$ depends upon the modeling assumptions and engineer preferences. Thus, there are $N$ sets of predictions $p$ in $M_{0}$.

The algorithm maintains a collection of model subsets $M$. In the first iteration, $M$ contains only $M_{0}$. During each iteration, the algorithm finds the biggest subset $M_{\max }$ in collection $M$ and chooses a sensor location $i$ that best separates the model predictions in $M_{\max }$. The algorithm finds location $i$ as follows. At each possible sensor location, a histogram containing predictions in $M_{\max }$ is built. Each histogram provides the number of models whose predictions lie within each interval. The size of intervals is determined for each measurement with the accuracy of the sensor $E_{\text {meas }}$ combined with the accuracy of the finite element model $E_{\text {model }}$. These values are provided to the algorithm as input. The number of intervals $N i$ is obtained by dividing the spread in the response of the model set by the sum of sensor and model accuracy.

The result of this operation is then rounded to the next larger integer. The histograms are built using $N i$ intervals starting at the lowest bound of the initial model set responses and each having a width equal to the sum of $E_{\text {meas }}$ and $E_{\text {model }}$. To measure the variability of model predictions at a given location, Robert-Nicoud et al. (2005) used the notion of entropy. The expression used to calculate entropy is the Shannon's entropy function (Shannon and Weaver 1949). Shannon's entropy function is a mathematical representation for the uncertainty in a set. For a random variable $X$, the entropy $H(X)$ is given by the following equation.

$$
H(X)=-\sum_{i=1}^{|X|} p_{i} \cdot \log \left(p_{i}\right)
$$

$p_{i}$ are the probabilities of the $|X|$ different possible values of $X$. For practical purposes, $0 \cdot \log (0)$ is taken to be 0 . Entropy is a measure of disorder in a distribution. The entropy for a given sensor location is calculated for the histogram of model 
predictions (the histogram being the discretization process). The probability $p_{i}$ of an interval is the ratio of the number $N_{i}$ of models in the interval by the total number of models $N_{\text {tot }}$. Equation 1 comes from the field of information theory. The disorder, and therefore entropy, is at maximum when model predictions show wide dispersion. At the best measurement locations, model predictions should have maximum variation. The algorithm iteratively chooses the locations with highest entropy for sensor placement. The algorithm stops when there is no further improvement to the maximum number of non-identifiable models, i.e., $M_{\max }$ does not become smaller.

\section{Case Study}

The algorithm is tested on a single-span composite truss bridge. Design models are usually based on conservative assumptions, and they contain a low level of detail. This way of proceeding has proved over time to lead to safe and economical structures. However, in order to perform system identification, the real structure must be represented as accurately as possible. Reducing modelling errors by including all structural features of the bridge decreases interval sizes, thus increasing the differentiability between candidate models. In addition to the main structural elements, the simulated bridge includes features such as sidewalk, security barrier, formwork haunch and pavement. The slope for water drainage is also included to capture its effect on load distribution. Since our case study is symmetric about its longitudinal and transverse axes, only a quarter of the bridge is modelled. Figure 1 shows a quarter of the bridge as analysed in ANSYS.

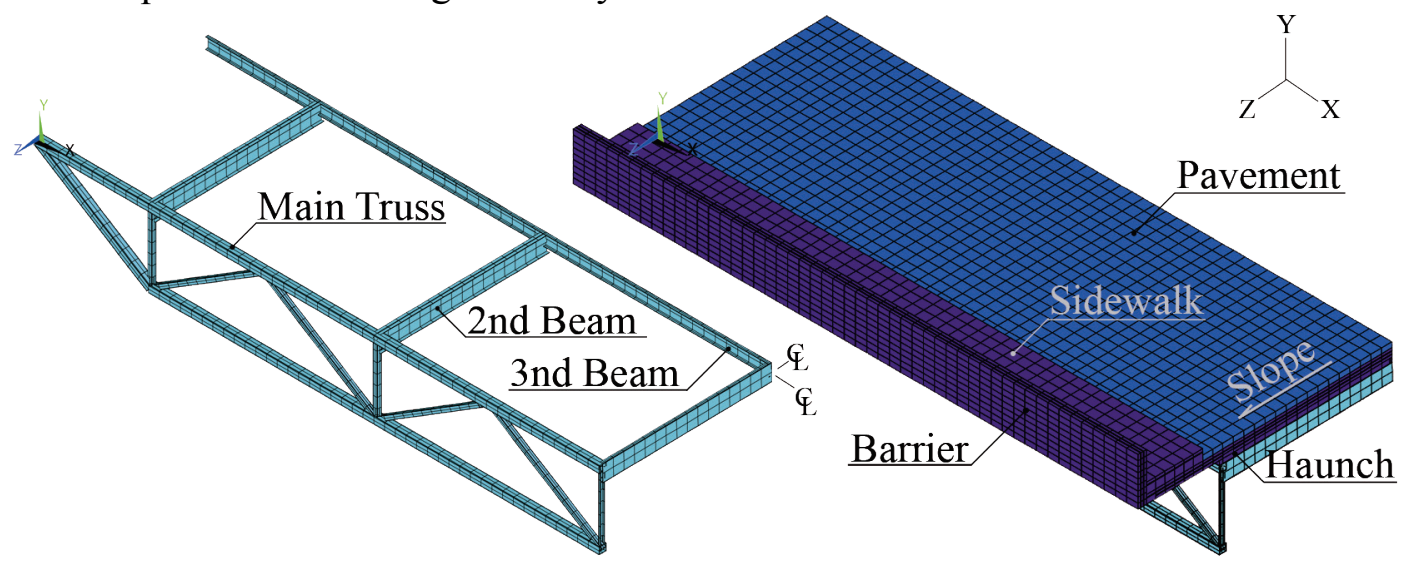

Figure 1 - Finite element model used in case study

In order to identify potential damage that may occur on the structure, several damage scenarios are generated. Three possible damage types are studied. The first possibility is a malfunction of an abutment that would restrain its free longitudinal movement. The second takes into account the development of fatigue cracks in main truss assemblies. Finally, the third possibility involves main-truss corrosion. As mentioned earlier, damage scenarios can be a combination of these possibilities and each may happen with a particular magnitude. Table 1 presents a summary of damage-scenario parameters. Every parameter is sampled according to a uniform probability density function. The range and unit of every parameter is also presented in Table 1. 
Table 1 -Parameters for damage-scenario model generation

\begin{tabular}{rlcc}
\hline Damage Type & Parameter & Range & Units \\
\hline Abutment locking & Number of affected abutment & {$[0-1]$} & - \\
& Stiffness of the abutment & {$[1-1 \mathrm{E} 6]$} & $\mathrm{N} / \mathrm{mm}$ \\
\hline Assembly cracking & Number of affected assemblies & {$[0-3]$} & - \\
\hline Main-truss corrosion & Number of affected zones & {$[0-3]$} & - \\
& Size of each affected zone & {$[500-1500]$} & $\mathrm{mm}$ \\
& Position of each affected zone & $\mathrm{X}:[0-\mathrm{L}]$ & - \\
& Intensity of the damage & {$[1-\mathrm{h}]$} & $\mathrm{mm}$ \\
\hline
\end{tabular}

For the purpose of this case study, 10,000 damage-scenario models are generated from combinations of values in Table 1 . The response of each model is evaluated for a predetermined set of possible sensor types and locations. The sensors used in this study are measuring displacement about the " $Y$ " axis, strains along the " $\mathrm{X}$ " or " $\mathrm{Z}$ " axis or rotation around "Z" axis. Axis "X", "Y" and "Z" are oriented in the global coordinate system as shown in Figure 1. Figure 2 shows the possible locations for each sensor type. The algorithm has to choose from a total of 34 measurement options using 3 sensors types positioned over 20 different locations along transverse and longitudinal axis ( $\mathrm{T}$ and $\mathrm{S}$ ). Table 2 presents the precision of sensors and model, used for this study.

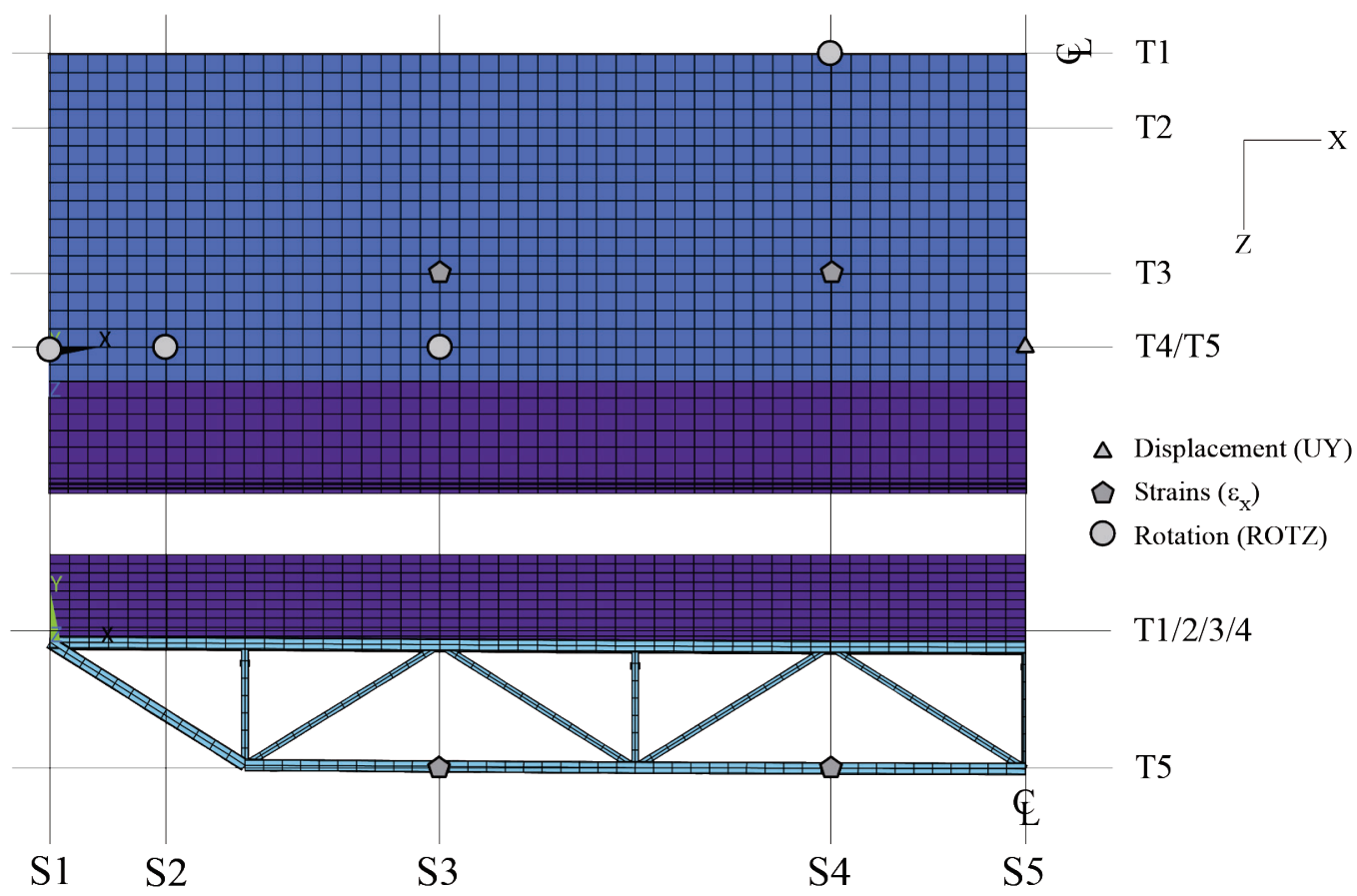

Figure 2 - Initial set of sensors (plan and elevation views) 
Table 2 - Sensor and model accuracy

\begin{tabular}{rlc}
\hline Device & Accuracy & Units \\
\hline Inclinometers $(\mathrm{ROTZ})$ & 3.0 & $\mu \mathrm{rad}$ \\
Strains $\left(\varepsilon_{\mathrm{x}} \& \varepsilon_{\mathrm{z}}\right)$ & 4.0 & $\mu \varepsilon$ \\
Displacement $(\mathrm{UY})$ & $2.5 \mathrm{E}-02$ & $\mathrm{~mm}$ \\
Finite Element Model & 5.0 & $\%$ \\
\hline
\end{tabular}

\section{Results}

Results from the 10,000 simulations are used to determine the optimal sensor locations. As shown in Figure 3, the algorithm stops choosing sensors when there is no further decrease in the number of non identifiable models. On the principle of greedy algorithm, each new sensor is added to the current set. It is interesting to note from the results presented in Figures 3 and 4 that no strain sensor along the " $Z$ " axis is selected, and that only one displacement sensor is selected. These results are expected, since the values of displacement and strains $\left(\varepsilon_{\mathrm{z}}\right)$ are small compared to the measurement error. In order to get useful data from these sensors, it would be necessary to increase the discriminatory characteristics of these two measurement quantities. This can be done by, for example, choosing more accurate measurement devices.

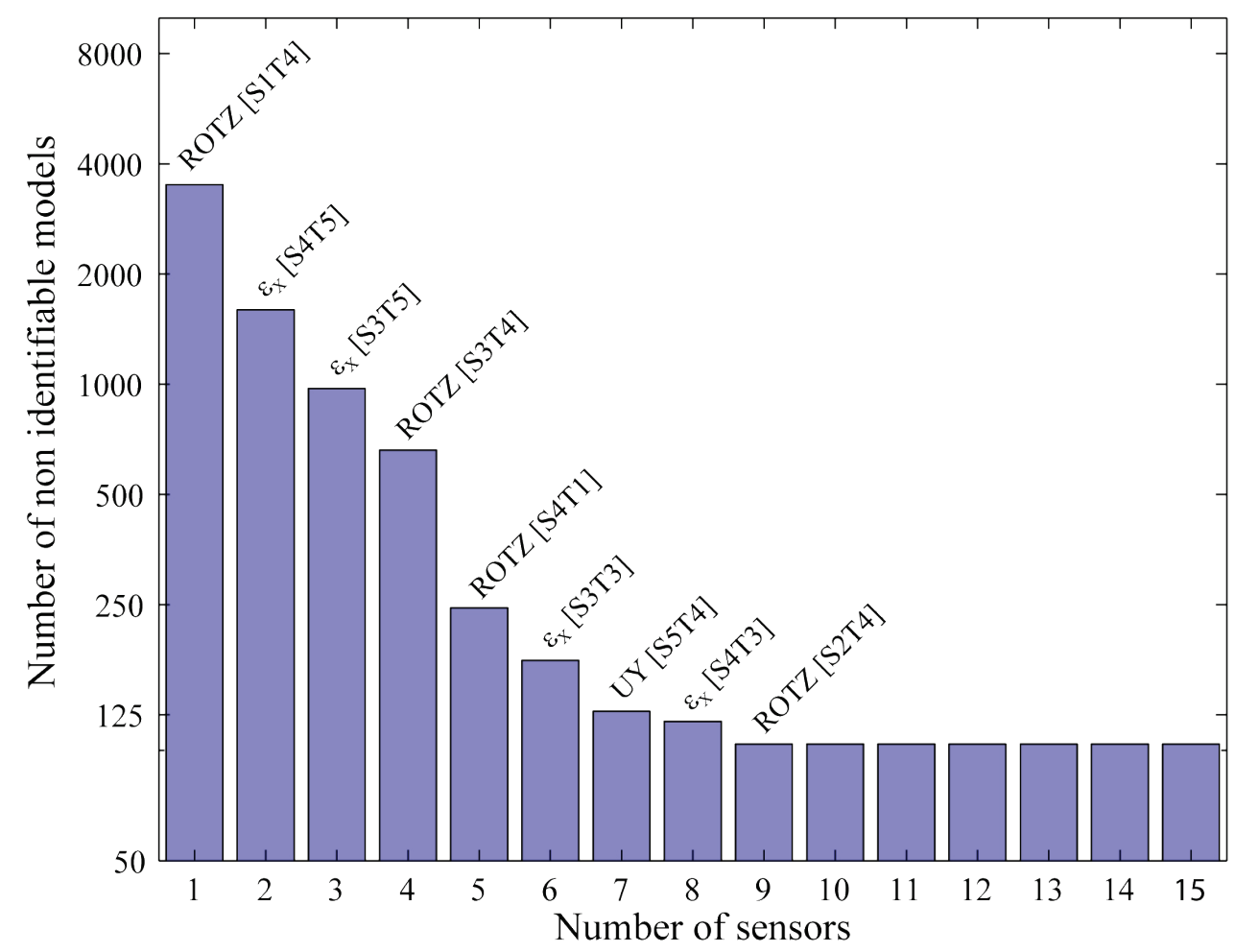

Figure 3 - Sensor placement evolution. The labels at the top of each bar refer to the sensor that is added. 
Through adopting the sensor configuration that is proposed by the algorithm, the worst case leads to a reduction of the initial subset of candidate models by a factor of almost 100 . This case represents the situation where the measurement data results in filtering to the subset with the largest number of candidate models, as presented in Figure 3. Figure 4 shows the final configuration of sensors.

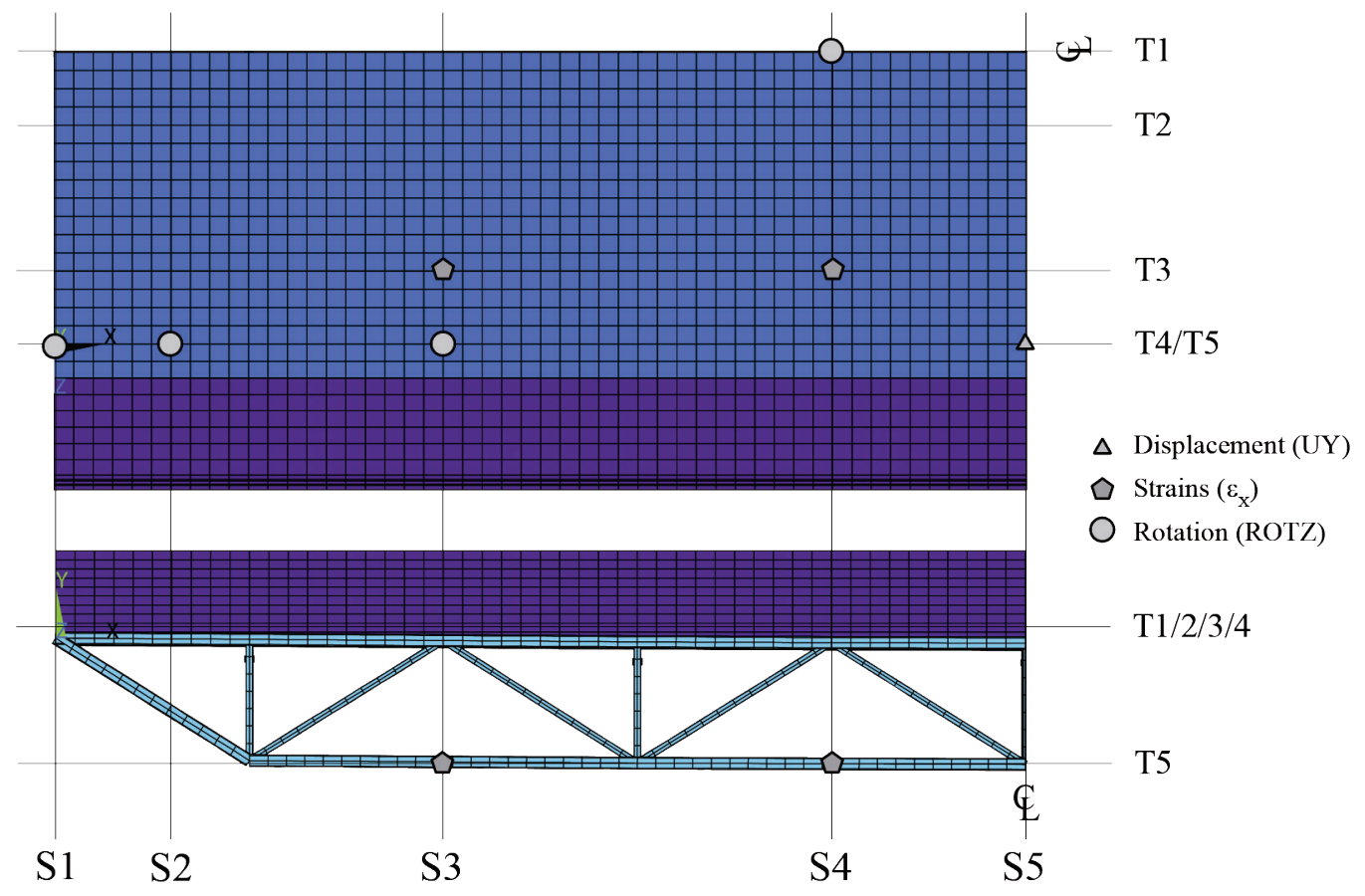

Figure 4 - Final sensor configuration

\section{Conclusion}

The conclusions of this research are:

- As an alternative to selecting and placing sensors using engineering experience alone, a more scientific approach based on sensor characteristics and modelling error is feasible.

- During sensor placement, considering different sensor types along with their characteristics enable selection of measurements that reduce most effectively the number of candidate models.

- In the case study presented in this paper, adding more than 9 sensors, from a possible set of 34, will not likely provide further useful information to identify the right behaviour from the models that are generated.

Future work will focus on applying the method with a global optimisation approach, in order to compare it with the currently implemented greedy algorithm. Another application of the method will involve extending the approach to behaviour identification of bridges. Measurements have been taken on a new, exceptionally slender bridge currently under construction in the city of Lucerne (Switzerland). 
Also, further work involves determining the effect of modelling error on sensor configuration.

\section{Acknowledgements}

This research is funded by the Swiss National Science Foundation under contract no. 200020-117670/1.

\section{References}

Banan, M. R., and Hjelmstad, K. D. (1994a). "Parameter-Estimation of Structures From Static Response .1. Computational Aspects." Journal of Structural Engineering-Asce, 120(11), 3243-3258.

Banan, M. R., and Hjelmstad, K. D. (1994b). "Parameters-Estimation of Structures From Static Response .2. Numerical-Simulation Studies." Journal of Structural Engineering-Asce, 120(11), 3259-3283.

Beck, J. L., and Katafygiotis, L. S. (1998). "Updating Models and Their Uncertainties. I: Bayesian Statistical Framework." Journal of Engineering Mechanics, 124(4), 455-461.

Bell, E. S., Sanayei, M., Javdekar, C. N., and Slavsky, E. (2007). "Multiresponse parameter estimation for finite-element model updating using nondestructive test data." Journal of Structural Engineering-Asce, 133(8), 1067-1079.

Catbas, F. N., Susoy, M., and Frangopol, D. M. (2008). "Structural health monitoring and reliability estimation: Long span truss bridge application with environmental monitoring data." Engineering Structures, 30(9), 2347-2359.

Domer, B., Raphael, B., Shea, K., and Smith, I. F. C. (2003). "A study of two stochastic search methods for structural control." Journal of Computing in Civil Engineering, 17(3), 132-141.

Garrett, J. H., Akinci, B., Matthews, S., Gordon, C., Wang, H. J., and Singhvi, V. "Sensor data driven proactive management of infrastructure systems." 13th International Workshop of the European-Group-for-Intelligent-Computing-inEngineering, Ascona, Switzerland, 262-284.

Jang, J. H., Yeo, I., Shin, S., and Chang, S. P. (2002). "Experimental investigation of system-identification-based damage assessment on structures." Journal of Structural Engineering-Asce, 128(5), 673-682.

Kang, F., Li, J.-j., and Xu, Q. (2008). "Virus coevolution partheno-genetic algorithms for optimal sensor placement." Advanced Engineering Informatics, 22(3), 362-370.

Katafygiotis, L. S., and Beck, J. L. (1998). "Updating Models and Their Uncertainties. II: Model Identifiability." Journal of Engineering Mechanics, 124(4), 463-467.

Kripakaran, P., Ravindran, S., Saitta, S., and Smith, I. F. C. "Measurement System Design Using Damage Scenarios." ASCE International Workshop on Compution in Civil Engineering, 615-623. 
Kripakaran, P., Saitta, S., Ravindran, S., and Smith, I. F. C. (2007b). Optimal sensor placement for damage detection: Role of global search, Ieee Computer Soc, Los Alamitos.

Ljung, L. (1999). "System Identification Theory for User." P. Hall, ed., New Jersey.

Meo, M., and Zumpano, G. (2005). "On the optimal sensor placement techniques for a bridge structure." Engineering Structures, 27(10), 1488-1497.

Morassi, A., and Tonon, S. (2008). "Dynamic Testing for Structural Identification of a Bridge." Journal of Bridge Engineering, 13(6), 573-585.

Nowak, A. S., and Cho, T. (2007). "Prediction of the combination of failure modes for an arch bridge system." Journal of Constructional Steel Research, 63(12), 1561-1569.

Raphael, B., and Smith, I. (1998). "Finding the right model for bridge diagnosis." Artificial Intelligence in Structural Engineering in Computer Science, Springer, Heidelberg, 308-319.

Raphael, B., and Smith, I. F. C. (2003). "Computer-Aided Engineering." Wiley, 306.

Robert-Nicoud, Y., Raphael, B., and Smith, I. F. C. (2005). "Configuration of measurement systems using Shannon's entropy function." Computers \& Structures, 83(8-9), 599-612.

Sanayei, M., Bell, E. S., Javdekar, C. N., Edelmann, J. L., and Slavsky, E. (2006). "Damage localization and finite-element model updating using multiresponse NDT data." Journal of Bridge Engineering, 11(6), 688-698.

Sanayei, M., Imbaro, G. R., McClain, J. A. S., and Brown, L. C. (1997). "Structural model updating using experimental static measurements." Journal of Structural Engineering-Asce, 123(6), 792-798.

Sanayei, M., and Saletnik, M. J. (1996). "Parameter estimation of structures from static strain measurements .1. Formulation." Journal of Structural Engineering-Asce, 122(5), 555-562.

Shannon, C., and Weaver, W. (1949). "The Mathematical Theory of communication." U. o. i. Press, ed.

Yeo, I., Shin, S., Lee, H. S., and Chang, S. P. (2000). "Statistical damage assessment of framed structures from static responses." Journal of Engineering Mechanics-Asce, 126(4), 414-421. 